\title{
EFFICACY AND SAFETY OF TREATMENT OF HYPOSECRETORY DRY \\ EYE WITH RICH-PLATELET PLASMA
}

Running head: Artificial tears vs. platelet rich plasma in dry eye

\author{
Víctor García-Conca $\mathrm{MD}, \mathrm{PhD}^{1}$ \\ Marta Abad-Collado BQ, $\mathrm{PhD}^{2}$ \\ Jose R Hueso-Abancens MD, $\mathrm{PhD}^{1}$ \\ Encarna Mengual-Verdú MD, $\mathrm{PhD}^{1}$ \\ David P Piñero, $\mathrm{PhD}^{3}$ \\ Fernando Aguirre-Balsalobre $\mathrm{MD}, \mathrm{PhD}^{1}$ \\ Julio C Molina MD ${ }^{1}$
}

From:

${ }^{1}$ Department of Ophthalmology. San Juan University Hospital, Alicante, Spain

${ }^{2}$ Mabad Laboratories, Alicante, Spain

${ }^{3}$ Department of Optics, Pharmacology and Anatomy. University of Alicante, Alicante,

Spain

\section{Corresponding authors:}

\section{Víctor García-Conca}

Department of Ophthalmology

San Juan University Hospital

Ctra. Nnal. 332, s/n 
03550 Sant Joan d'Alacant, Alicante, Spain

e-mail: viconca@live.com

\author{
David P. Piñero
}

Department of Optics, Pharmacology and Anatomy.University of Alicante.

Crta San Vicente del Raspeig s/n 03016

San Vicente del Raspeig, Alicante, Spain

Tel: +34-965903500, Fax: +34-965903464

e-mail: david.pinyero@ua.es

\title{
Financial disclosure
}

The author David P Piñero has been supported by the Ministry of Economy, Industry and Competitiveness of Spain within the program Ramón y Cajal, RYC-2016-20471. The authors have no proprietary or commercial interest in the medical devices that are involved in this manuscript. 


\begin{abstract}
Objective: To evaluate and compare the symptomatology and clinical findings in hyposecretory dry eye of the treatment with platelet rich plasma (PRP) and artificial tears of sodium hyaluronate $(\mathrm{SH})$.

Methods: Blind single-center prospective comparative randomized study including 84 patients with hyposecretory dry eye and mean age of 64.0 years. Two groups were differentiated depending on the treatment applied: 44 patients treated with PRP (PRP group), and 39 patients treated with artificial tears of $\mathrm{SH}$ ( $\mathrm{SH}$ group). Changes in Schimer test, tear osmolarity, corneal and conjunctival staining, tear film break-up time (TF-BUT), conjunctival hyperemia, dry eye-related symptoms with the OSDI questionnaire, and caliciform cell density were evaluated during 30 days of treatment.

Results: Significantly larger reduction of symptomatology $(\mathrm{p}<0.001)$, visual improvement $(\mathrm{p}<0.001)$, reduction of hyperemia $(\mathrm{p}<0.001)$ and corneal and conjunctival staining $(p<0.001)$, increment of Schirmer test outcome $(p \leq 0.005)$ and reduction of osmolarity were found in the PRP group in both eyes compared to SH group at 15 and 30 days of treatment. Likewise, a significantly higher increment of caliciform cell density $(\mathrm{p}<0.001)$ was found in the PRP group. Strong and statistically significant correlations were found in the PRP group of the change achieved in visual acuity, hyperemia, osmolarity, and conjunctival and corneal staining with the baseline values of these variables $(\mathrm{p}<0.001)$.

Conclusions: PRP treatment in hyposecretory dry eye induces a more significant positive effect over symptomatology and different dry eye signs than $\mathrm{SH}$, especially in moderate and severe cases.
\end{abstract}




\section{INTRODUCTION}

The use of eye drops prepared using blood products in the ocular surface was initially described by Ralph and Doane (1975), showing the efficacy of the autologous serum (AS) for the treatment of keratoconjunctivitis sicca. More than 20 years after, more studies reported the benefits of AS in the management of the dry eye associated to the Sjögren's syndrome and persistent epithelial defects (Fox et al 1984). Since then, the interest in the use of hematological-derived substances for topical use as a treatment approach for ocular surface disorders has increased significantly in the last years (Alió \& Rodríguez 2015; Anitua \& Muruzabal 2016), with a great variety of studies reported (Alió et al 2017; Anitua E 2015; Avila 2014; Celebi et al 2014; Javaloy et al 2013; Panda et al 2012; Urzua et al 2012; López-Plandolit et al 2011; Marquez De Aracena Del Cid R 2009; Alió et al 2007; Noda-Tsuruya \& Asano-Kato 2006; Kojima et al 2005; Noble \& Loh 2004; Tananuvat \& Daniell 2001). Among these substances, the use of platelet rich plasma (PRP) has been extensively investigated for the treatment of several ocular surface conditions (Alió et al 2017; Anitua 2015; Avila 2014; Javaloy et al 2013; Panda et al 2012; López-Plandolit et al 2011; Marquez De Aracena Del Cid R 2009; Alió et al 2007). PRP can be defined as a volume of AS containing a platelet concentration over the basal level (150.000-350.000/ $\mu 1)$ (Wroblewski et al 2010). The therapeutic effect of PRP has been demonstrated for the treatment of dry eye disease (Avila 2014; López-Plandolit et al 2011; Alió et al 2007), persistent epithelial defects (Ronci \& Ferraro 2015; Nishida \& Nakamura 1996), graft-versus-host disease (Pezzotta \& Del Fante 2012), chemical burns (Egbert et al 1977), corneal ulcers and perforations (Alió et al 2013b; Alió \& Rodríguez 2013), post-LASIK chronic ocular surface 
syndrome (Alió et al 2017), recurrent corneal erosions (Lee et al 2016; del Castillo et al 2002), Mooren's ulcer (Sangwan et al 1997), superior limbic keratoconjunctivits (Goto et al 2001), and neurotrophic keratopathy (Matsumoto et al 2004).

Concerning the application of PRP for the management of dry eye, a successful result with minimal complications has been reported in three observational studies (Avila 2014; López-Plandolit et al 2011; Alió et al 2007). Avila et al (2014) treated 4 patients with severe dry eye syndrome due to a lachrymal gland dysfunction by means of injections of $1 \mathrm{ml}$ of PRP (0, 4, 8 and 12 weeks). In all cases, a significant improvement was observed in the results of the Schirmer test, lissamine green staining test (modified Oxford scale: $8.0 \pm 0.6$ to $2.8 \pm 0.5$, $\mathrm{p}<0.005$ ), and tear film break-up test (TFBUT) $(4.3 \pm 0.4$ to $12.3 \pm 0.7 \mathrm{~s}, \mathrm{p}<0.005) .{ }^{7}$ Alió and colleagues $(2007)$ conducted another observational study in which 18 patients with different grades of dry eye disease were treated with PRP applied 4-6 times a day for 1 month. These authors found an improvement of 1 or more lines of visual acuity in $28 \%$ of patient without worsening in the rest, an improvement of 2 seconds in $50 \%$ of the tear film brak-up time (TF-BUT) without worsening in the rest, an improvement in conjunctival hyperemia in $86 \%$ of eyes, and a statistically significant improvement in conjunctival impression cytology outcomes. Regarding the observational study of López-Plandolit et al (2011), it shows the results obtained in 16 patients with moderate-severe dry eye disease after treatment with PRP, finding a significant improvement in the symptom questionnaire $(\mathrm{p}<0.001)$, but without a statistically significant reduction in the degree of metaplasia observed in conjunctival impression cytology. However, in spite of these positive outcomes, there are no controlled comparative studies demonstrating the superiority of PRP treatment in dry eye disease over conventional treatment with artificial tears. It is assumed that the PRP provides more benefit than artificial tear eye drops as PRP has a more similar 
composition compared to the natural tear in addition to the regenerative effect of its concentration of growth factors, but there are no comparative studies confirming this. According to the Dry Eye WorkShop (DEWS) II report (Jones et al 2017), PRP belongs to the most powerful therapeutic strategies in dry eye disease. This level of therapies, which comprises autologous serum and ombilical cord serum as well, corresponds to the step 3 in the treatment scale. The step 3 is recommended to be used when step 2 and step 1 treatment are inefficient. However, the authors of DEWS II report stated that the management algorithm presented in their report did not represent a rigid stepwise approach, but rather should be viewed as an organizational tool to use when initiating treatment of DED, to select interventions likely to provide most benefit. In our clinical practice, treatments are not based on steppened recommendations, but rather on treating those aspects of the pathophysiology that are the cause of DED initiation and chronification. If a treatment provides a more efficient recovery of a condition without relevant side effects, it should be used as a primary option or the patient should be informed at least of it. Among the factors involved in the pathophysiology of DED according to DEWS II (Bron et al 2017) are tear inestability, tear hyperosmolarity inflammation and tissue damage. Our aim was to compare two different treatments, artificial tear vs. PRP, in order to compare the level of influence of these two therapeutic options on these processes and therefore to confirm the potential superiority of PRP over artificial tears in hyposecretory dry eye. Specifically, our aim was to evaluate and compare the clinical outcomes in terms of visual acuity, conjunctival hyperemia, tear film production and stability, osmolarity and symptomatology of the treatment with PRP and artificial tears of sodium hyaluronate in patients with hyposecretory dry eye. 


\section{MATERIAL AND METHODS}

\section{Patients}

A total of 84 patients with hyposecretory dry eye and a mean age of 64.0 years were included in this blind single-center prospective comparative randomized study. All participants were selected from the ocular surface section of the Department of Ophthalmology and from the Department of Reumathology of San Juan Hospital (Alicante, Spain), where this investigation was developed in the period from January 2013 to November 2014. Two groups were differentiated depending on the treatment applied: PRP group, including 44 patients treated with PRP, and SH group, including 39 patients treated with artificial tears of a hypotonic aqueous solution of sodium hyaluronate $(\mathrm{SH})$ containing a number of important ions (Vismed, TRB Chemedica, UK). All patients were informed about the study and gave their informed consent to perform the measurements following the tenets of the Declaration of Helsinki. The study was approved by the Ethics Committee of the San Juan Hospital.

Inclusion criteria for the study were age between 18 and 90 years, Schirmer test outcomes of $5.5 \mathrm{~mm}$ or lower, presence of dry eye symptoms evaluated with the OSDI (Ocular Surface Disease Index) questionnaire (OSDI $\geq 13$ ), and presence of corneal of corneal and conjunctival staining (score $\geq 1$, Oxford scale). Exclusion criteria were previous keratorefractive surgery, previous cataract surgery, active ocular surface disease except dry eye, eyelid alterations complicating blinking, TF-BUT below 10 seconds, topical ocular treatment, antecedents of mucocutaneous disease affecting the ocular surface, antecedents of ocular surface caustication, contact lens wearers, antecedents of herpetic keratitis, and presence of contraindications for being PRP donor. 


\section{Clinical protocol}

Patiens were instructed not to use topical treatment 48 hours before first visit. An initial evaluation of the dry eye-related symptoms with the OSDI (Ocular Surface Disease Index) questionnaire. A comprehensive ophthalmologic baseline examination was performed in all patients following the same order of procedures with recovery time between them. These examination comprising uncorrected and best-corrected visual acuity, manifest refraction, osmolarity measurements (one on each eye, if interocular differences $\geq 10$ units, the measurement was repeated and mean value calculated)(Tear Lab, TearLab Corp, San Diego, CA, USA), slit-lamp biomicroscopy examination. On slit lamp examination, the level of hyperemia was evaluated by using the Brien Holden Vision Institute scale (score from 0 to 3) (Baudouin et al 2015). TF-BUT measurement (three consecutive measurements), evaluation of corneal and conjunctival staining (Fluo-Plus strip, Gecis, Ecoparc, Domaine de Villemorant, France), Schimer test (Schirmer-Plus strip, Gecis, Ecoparc, Domaine de Villemorant, France) (closed eyes during 5 minutes without anesthesia), and conjunctival impression cytology (CIC). All measurements were performed by the same single experienced examiner (VGC). Likewise, the TF-BUT was evaluated after applying the Fluo-Plus strip in the inferior bulbar conjunctiva and observing the tear film using the blue filter of the slit lamp. Three consecutive measurements of TF-BUT were obtained and the mean value calculated. Regarding the level of corneal and conjunctival staining, it was also evaluated after the application of the fluorescein strip using the "cobalt" filter of the slit lamp and a Kodak Wratten 12 barrier filter. Corneal and conjunctival (mean value of temporal and nasal zones) staining were graded between 0 and 5 according to the Oxford scale (Bron et al 2003). 
The last test of the baseline examination was the performance of CIC. For this purpose, a drop of sterile 5\% lidocaine anesthetic without preservatives was instilled before beginning with the measurement process. The plastic projections of a Biopore membrane (Millipore ${ }^{\circledR}$ PICM01250, Merck KGaA, Darmstadt, Germany) were removed with scissors in order to avoid the erosion of the conjunctiva. It was applied on the inferior temporal conjunctiva away from the area used for the position of the Schirmer strip (interpalpebral area). In our experience, the use of this location is associated to less patient discomfort. For this reason, the patient was asked to look towards nasal-superior direction during CIC. Cytology was performed only on the eye with the highest score of conjunctival staining in the area evaluated. The Biopore membranes were sent to the anatomical pathology department for processing according to this protocol: fixation with $96 \%$ ethanol, distilled water 5 minutes, periodic acid $1 \%$ (carlo erba) for 5 minutes, washing with distilled water, Schiff's reagent (carlo erba, 1: 3 in distilled water) for 2 minutes, washing with distilled water for 5 minutes, hematoxylin for 2 minutes, $1 \%$ ammonia water for 2 minutes, dehydration with increasing concentrations of ethanol $70 \%$ for 2 minutes and $96 \%$ for 1 minutes, rinse with xylol for 5 minutes (3 times), and mount on glass slides with Entellan.

Once finished the baseline examination, inclusion and exclusion criteria were revised. If inclusion criteria were accomplished and any of the exclusion criteria were present, the patient was assigned randomly (random number sequence) to the $\mathrm{SH}$ or PRP group. If the patient was assigned to the SH group, four eye drop bottles containing SH $0.18 \%$ (osmolarity $150 \mathrm{mOsm} / 1$, molecular weight $1.2 \mathrm{MDa}$ ) were provided. If the patient was assigned to the PRP group, a sample of blood (40 ml) was extracted and PRP eye drops were prepared following this procedure: 
1.- Blood processing performed with the RegenKit ${ }^{\circledR}$ Ophthalmology PRPTM Preparation kit (Regen Lab USA LLC, New York, USA)

2.- Once filled the blood tubes, they were inverted several times with care.

3.- The centrifuge (LABOFUGE 400) was balanced by filling the counterweight and a centrifuging force of $1500 \mathrm{~g}$ was applied for 5 minutes.

4.- The tubes were then inclined to resuspend the cell pellet in the supernatant to obtain $4.5 \mathrm{ml}$ of PRP per tube.

5.- Under a laminar flow hood (Nüve LN 120, Nuve, Ankara, Turkey), the AS with the platelet concentrate was finally transferred to four eye drop dispensing canisters (osmolarity: $316.23 \pm 6.31 \mathrm{mOsm} / \mathrm{l} ; \%$ platelet per ml: $536.53 \pm 202.210^{3} / \mu \mathrm{l}$; concentration increase: $x 2.88 \pm 0.69)$.

Each patient from the PRP group was provided with a means of transport to maintain the cold chain until they reach home. They were instructed to keep the bottle used during the week at the refrigerator door $\left(4^{\circ} \mathrm{C}\right)$ whereas the rest was kept in the freezer. Patients should apply eye drops every 3 hours from 8:00 a.m. to 23:00 p.m., estimating a total of 6 applications a day.

Once prescribed the treatment, a second and third visit was performed at $14 \pm 2$ and $28 \pm 2$ days after its beginning. The same tests than in the baseline visit were performed, except CIC that was only performed in the second visit. In case of noncompliance in more than $30 \%$ ( $\leq 4$ applications/day) of the estimated applications per day, the patient was excluded from the study. Side effects if present were registered after each visit. 


\section{Statistical analysis}

For an appropriate design of the study, a sample size calculation was performed before recruiting patients. Assuming a confidence level of $95 \%$, accuracy of $4 \%, \alpha$ error of $5 \%$ and percentage of losses of $10 \%$, a sample size of 92 patients was recommended. Finally, a total of 90 patients were included in the study. At the end of the study, 7 patients who did not attend to some scheduled visits or did not comply with the treatment guidelines were excluded. The final number of patients included in the statistical study was 84 (164 eyes). We analyzed the data of each eye of the same subject separately in order to avoid the potential bias of analyzing the combined data from fellow eyes of the same individual that are significantly correlated.

The statistical analysis was performed using the software SPSS version 15.0 for Windows (IBM, Armonk, NY, USA). Normality of all data distributions was confirmed by means of the Kolmogorov-Smirnov test. A descriptive study was performed for the data of the two study groups in the different visits of the follow-up, expressed as mean, standard deviation (SD), median and range. Besides this, the Levene test was used to assess the equality of variances. If the analyzed samples followed a normal distribution, the Student $\mathrm{t}$ test was used for paired data to analyze changes occurring between consecutive visits, while the Wilcoxon test was used if the data distributions did not follow normality. For the comparison between groups of treatments, the Student $t$ test for unpaired data was used if parametric statistics could be applied and the MannWhitney test otherwise. 


\section{RESULTS}

A total of 168 eyes of 84 patients with an age ranging from 36 to 90 years old were included in the study (mean: 64.0; standard deviation, SD: 11.2; median: 67.0 years). The sample was comprised of 81 females (96.4\%) and 3 men (3.6\%). Sjögren syndrome was the most frecuent risk factor associated to these patients $(41 \%$ in PRP group $/ 31 \%$ in $\mathrm{SH}$ group). Table 1 summarizes the baseline values in $\mathrm{SH}$ and PRP groups. As shown, there were significant differences between groups at the baseline in terms of right $(p=0.002)$ and left eye hyperemia $(p=0.001)$, Schirmer test value in right eye $(p=0.022)$, level of corneal and conjunctival staining in both eyes $(p<0.001)$, and in the result of CIC $(p=0.007)$. In all cases, the worst outcome was present in the PRP group. Considering that both groups were not completely matched at the baseline, we analyzed the results in each group and performed comparisons in terms of variations in each parameter evaluated, as displayed in Tables 2 and 3.

\section{Comparison among PRP and SH groups}

At 15 days after beginning the treatment (Table 2), a significantly larger reduction of the OSDI score was observed in the PRP group compared to the SH group $(\mathrm{p}=0.001)$ (Figure 1), as well as a more remarked visual improvement in both eyes $(p<0.001)$, a higher reduction in the level of hyperemia in both eyes $(p=0.001)$, a larger increment of the outcome of the Schirmer test in both eyes $(\mathrm{p} \leq 0.002)$, a larger increment of TF-BUT values $(\mathrm{p}=0.003)$ in the left eye, a higher reduction of osmolarity in both eyes $(\mathrm{p}=0.003)$ (Figure 2$)$, and a more significant reduction of corneal and conjunctival stanining levels $(\mathrm{p}<0.002)$ in both eyes. At 30 days after beginning the treatment (Table 3), the same trends were observed for OSDI score $(p=0.001)$, visual 
improvement in both eyes $(p<0.004)$, the level of hiperemia in both eyes $(p<0.002)$, the result of the Schirmer test in both eyes $(p \leq 0.005)$, and the level of corneal and conjunctival level in both eye $(\mathrm{p}<0.001)$. Likewise, significantly higher increments in TF-BUT but only in left eye $(p=0.016)$ and in the density of caliciform cells $(p<0.004)$ were found in the PRP group compared to the SH group.

\section{Changes and correlations in PRP group}

In PRP group, a significant reduction in OSDI score (15 and 30 days, $\mathrm{p}<0.001$ ), as well as significant visual improvement in both eyes ( 15 days: $\mathrm{p}<0.001 ; 30$ days: $\mathrm{RE}$ $\mathrm{p}=0.004$, LE $\mathrm{p}=0.002)$, improvement of the level of hyperemia (15 days: $\mathrm{p}<0.001 ; 30$ days: $\mathrm{RE} \mathrm{p}=0.003$, LE $\mathrm{p}=0.021$ ), reduction in tear osmolarity ( 15 days: $\mathrm{p}<0.003 ; 30$ days: $R E p=0.002$, LE $p=0.002$ ), increase in Schirmer outcome ( 15 days: $p<0.001 ; 30$ days: $R E p=0.003$, LE $p=0.001$, and reduction of the level of corneal and conjunctival staining (15 days: $\mathrm{p}<0.001 ; 30$ days: $\mathrm{p} \leq 0.002$ ) in both eyes were found. Likewise, a small significant increase in TF-BUT of left eye (LE) was observed at 15 days after beginning the treatment $(\mathrm{p}=0.002)$ and day $30(\mathrm{p}=0.016)$ but not significant increase in TF-BUT of right eye $(\mathrm{RE})(\mathrm{p}=0.62)$ at 15 days and $30(\mathrm{p}<0.47)$. A significant incremement was observed in the result of CIC when comparing baseline with the end of the follow-up $(\mathrm{p}=0.001)$.

Concerning the visual change in RE, visual acuity was found to be correlated with baseline (RE: $r=-0.701, p<0.001$; LE: $r=-0 .-65$, $p<0.001$ ). Furthermore, the change in hyperemia in both eyes was found to correlate with the baseline level of hyperemia (RE: $r=-0.765, p<0.001$; LE: $r=-0.692, p<0.001$ ) and the change in osmolarity with the baseline value of this parameter in both eyes (RE: $r=-0.714, p<0.001$; LE: $r=-0.752$, $\mathrm{p}<0.001)$. The change in conjunctival staining in both eyes was also found to be 
correlated with the baseline level of this sign (RE: $r=-0.707, p<0.001$; LE: $r=-0.685$, $\mathrm{p}<0.001)$ as well as the change in corneal staining with the baseline level in LE ( $\mathrm{r}=-$ $0.732, \mathrm{p}<0.001)$.

\section{Changes and correlations in SH group}

In $\mathrm{SH}$ group, a small but significant reduction in OSDI score was observed at 15 $(p=0.006)$ and 30 days of the follow-up $(p=0.001)$. Statistically significant changes in this group were only observed in the following variables: reduction in tear osmolarity at 15 days in both eyes (RE: $p=0.007$; LE: $p=0.039$ ), reduction of conjunctival staining in both eyes only at 15 days ( $\mathrm{p}=0.020$ ), and reduction of corneal staining in $\mathrm{RE}$ at 15 days $(p=0.020)$.

The change in conjunctival staining in LE was significantly correlated with baseline conjunctival staining $(\mathrm{r}=-0.549, \mathrm{p}=0.001)$. Likewise, the change in caliciform cell density measured by CIC was found to be correlated with baseline RE $(r=0.658$, $\mathrm{p}=0.008)$ and LE hyperemia level $(\mathrm{r}=0.716, \mathrm{p}=0.006)$, conjunctival staining in LE $(\mathrm{r}=0.683, \mathrm{p}=0.010)$, and corneal staining in $\mathrm{RE}(\mathrm{r}=0.656, \mathrm{p}=0.008)$ and $\mathrm{LE}(\mathrm{r}=0.757$, $\mathrm{p}=0.003)$.

\section{DISCUSSION}

The use of artificial tears still remains the most commonly used option of treatment in dry eye (Thulasi \& Djalilian 2017). However, an artificial tear is a pharmaceutical product that tries to simulate the properties of the true tears of our eyes in terms of water composition, fluidity, osmolarity, $\mathrm{pH}$ and surface tension, being far from resembling them (Drew et al 2018). It should be considered that the origin of the 
tear is the plasma in its passage through the different glands responsible for its production. Therefore, the similarity between plasma and tears is significant, being reasonable to think that they can have comparable effects on the ocular surface (Drew et al 2018). Some clinical studies have already demonstrated the superiority of AS versus artificial tears in terms of improvement of symptoms (OSDI), but with no statistically significant differences in corneal staining or TF-BUT (Urzua et al 2012). Likewise, Celebi et al (2014) also found statistically significant differences between AS and artificial tear in OSDI and TF-BUT, but not in Schirmer test result, and corneal and conjunctival staining. In comparison to AS, PRP provides a greater concentration of platelets and their release of growth factors. In the observational study of Alio et al (2007), an improvement was observed in patients with symptomatic dry eye treated using PRP in terms of symptoms, conjunctival hyperemia, corneal staining and CIC. These changes in corneal staining and CIC outcomes are related to a tissue regeneration that could be related to the regenerative benefit of PRP versus AS. López-Plandolit et al (2011) also found improvement in clinical slit lamp signs and Schirmer test, but without significant change in the level of conjunctival metaplasia. Considering all these studies and the fact that no controlled clinical trials have been conducted comparing the effect of PRP in hyposecretory dry eye versus artificial tears of SH, the performance of the current study was considered as necessary to elucidate the real benefit of PRP over conventional treatment with artificial tears.

In our sample, a significant reduction of the OSDI score was found with both type of treatments, although the magnitude of this change was significantly higher with the use of PRP. Therefore, a more significant level of control of symptomatology seems to be achievable with PRP. Furthermore, the improvement in symptoms was more significant during the first 2 weeks of treatment. The reason for this may be that the 
regenerative effect of PRP is more active in the first days of treatment due to the biological stability of the platelets and growth factors, which is affected by changes over time as well as by their way of conservation. Specifically, in our sample, at 15 days after treatment beginning, only 4 patients $(9 \%)$ in the PRP group referred no improvement in symptoms, whereas this percentage was almost $60 \%$ in the $\mathrm{SH}$ group. This significant improvement in symptomatology found in our series with PRP is comparable to that found in previous series, such as Alió et al (2007) and LópezPlandolit et al (2011) that registered reductions in the OSDI scores of $89 \%$ and $75 \%$, respectively. Likewise, other studies have demonstrated that more improvement in symptoms was achieved in patients treated with hematological derivatives against artificial tears, as those conducted by Celebi et al (2014) and Kojima et al (2005). Concerning SH, it should be noted that about $86 \%$ of patients had some type of artificial treatment treatment prior to inclusion in the study. In spite of this, patients treated with SH reported a symptom improvement of lesser extent. Indeed, in the PRP group, there were no reports of worsening symptoms on any of the days during the treatment, whereas $17 \%$ of patients in the SH group did show a worsening at 15 days after the beginning of the treatment.

Regarding visual changes, they were significantly lower in the $\mathrm{SH}$ group compared to PRP group. This more significant visual improvement is consistent with the significantly higher reduction of corneal staining and increase in mean TFBUT. Epithelial damage (Kaido et al 2011) and tear film instability (Beníez-Del-Castillo et al 2017) are factors that have been found to contribute to a visual deterioration in dry eye. However, some previous series have shown limited visual improvements in dry eye with the use of hematological derivatives. Alió and colleagues (2007) found in their series that only $28 \%$ of patients treated with PRP experienced a visual improvement of 
1 line or more. Noble \& Loh (2004) and Urzua et al (2012) did not find significant improvements in visual acuity in patients treated with autologous serum versus artificial tears. Several factors may account for the differences in the level of visual improvement achieved between studies such as the different number of patients included, the clinical protocols used, but especially differences in the percentage of cases with central involvement of the cornea. If the corneal epitelial defect is not located centrally, it should not have an influence on the level of visual acuity. In our study, the visual improvement in the PRP group was associated with a significantly higher reduction of corneal staining compared to SH group in spite of having higher levels of corneal staining at baseline those eyes treated with PRP. Specifically, after 15 days of treatment, corneal staining decreased by $50 \%$ in the PRP group, whereas this reduction was more limited in the SH group. This result is consistent with that reported by Alió et al (2007) who described an improvement in corneal staining by $72 \%$ in dry eyes treated with PRP. Kojima and colleagues (2005) also described a significant improvement in patients treated with autologous serum compared to those treated with a saline solution.

Besides improvements in corneal staining, changes in conjunctival staining and hyperemia were also observed in our sample. An improvement in the level of hyperemia was detected with both treatments, although it was significantly greater with PRP and especially after the first 15 days of treatment. There is a moderation of this improvement after the following 15 days. This result is similar to that found by Alió and coauthors (2007), who reported an improvement in the level of hyperemia by $86 \%$ in a group of patients with dry eye and treated with PRP. It should be noted that conjunctival hyperemia is the result of vascular vasodilation secondary to an increase in mediators of inflammation that are increased in certain phases of symptomatic dry eye (LópezMiguel et al 2016). In this sense, hyperemia can be considered as an indirect marker of 
inflammation of the ocular surface. Concerning conjunctival staining, a significantly higher reduction was also observed in the PRP group compared to $\mathrm{SH}$ group. Specifically, the improvement in staining after the first 15 days of treatment in the PRP group was almost two points on the Oxford scale, which represents a reduction by $50 \%$. In the following 15 days, an additional 50\% reduction was observed which in absolute values was equivalent to half a point of the Oxford scale. In contrast, the decrease in staining in $\mathrm{SH}$ was only statistically significant at 15 days of follow-up, and the magnitude of such decrease was markedly lower. This contrasts with previous studies evaluating conjunctival staining evaluated with other staining substances. LópezPlandolit et al (2011) found an improvement in conjunctival staining with lysamine in eyes treated with PRP, but this change did not reach statistical significance. In contrast, Kojima and coauthors (2005) did not find significant differences in conjunctival staining using Bengal rose between eyes treated with autologous serum and artificial tears.

As previously indicated, the aim of the current study was to evaluate and compare the effect of PRP and SH in patients with hyposecretory dry eye. This was done to exclude or minimize the potential influence of the evaporative mechanism and to obtain then more specific conclusions on the PRP effect on the clinic and physiopathology of dry eye. Specifically, in our sample, we evaluated the impact of treatments on tear film production by analyzing the outcomes obtained with the Schirmer test. In group PRP increase in the outcome obtained with the Schirmer test was obtained at 15 days of treatment, with a significant additional improvement at the end of the follow-up. In terms of changes, the improvement obtained with the PRP treatment was significnatly higher tan that obtained with SH. These differences among treatments are due to the higher regenerative capability of PRP and its effect on the acinar cells of the lacrimal glands (Avila 2014). Indeed, Avila (2014) reported 
significant improvements of the Schirmer test outcome after PRP injections close to the lacrimal gland. In contrast, no significant differences were found in clinical trials comparing autologous serum against artificial tears (Celebi et al 2014; Kojima et al 2005; Noble \& Loh 2004). It should be considered that the platelet concentration and the regenerative capability of autologous serum is lower.

Besides the impact of the two options of treatment on tear production, the effect on tear film stability has been also evaluated, which is in relation with the evaporative component of dry eye. For such purpose, the TF-BUT was measured and, as expected considering that we only included subjects with hyposecretory dry eye, changes in BUT were minimal and no statistically significant in most of cases. Indeed, TF-BUT was within the range of normality in all cases at baseline. Therefore, we could not establish the effect of PRP compared to SH on tear film stability as patients without alteration of this factor was selected for the study. Other studies comparing autologous serum versus artificial tear did not find either significant changes in TF-BUT (Celebi et al 2014; Alió et al 2007; Noble \& Loh 2004). In contrast, significant improvements in TF-BUT were reported by Avila (2014) in a group of subjects in which PRP injection was performed close to the lacrimal gland as well as in the clinical trial of Kojima et al (2005) comparing autologous serum and artificial tear.

Tear film osmolarity is one factor implicated in the physiopathological mechanisms of corneal epitelial damage and activation of inflammatory processes, being considered the best biomarker for dry eye disease (International Dry Eye Workshop, 2007). In our sample, at 15 days of treatment, PRP and SH were found to decrease tear osmolarity, although this reduction was significantly higher in those eyes using PRP. An additional significant decrease in osmolarity was only observed in the PRP group at 30 days of treatment. It is curious this ability of PRP of reducing tear 
osmolarity considering that this substance is hyperosmolar $(316.23 \pm 6.31 \mu \mathrm{OSM} / \mathrm{ml})$. This may be explained by the regenerative effect of PRP over epithelium, promoting the reduction of pro-inflammatory factors released and the restoration of homeostasis. This would also consistent with the significant reduction in the level of conjunctival hyperemia achieved with the treatment in our series. To our knowledge, this is the first study comparing PRP versus artificial tears in terms of osmolarity, confirming the great improvement achieved in this variable when using PRP.

An additional diagnostic test used in our study was CIC, wich is a technique providing information about anatomical-pathological features of the ocular surface. Different pathological conditions may affect the ocular surface, including dry eye. Specifically, in this condition, a significant reduction of the number of caliciform cells secretory of mucoproteins has been reported that affects to the stability of the tear film (Nelson \& Wright 1984). Specifically, this reduction in the cell counts conducted over the interpalpebral área has been found to be $17 \%$ in dry eye patients (Amparo \& Wang, 2013). In our sample, we found with PRP treatment a significant increase in caliciform cell density assessed by CIC. Again, as with most of the rest of variables evaluated, the increase in the caliciform cell density was significantly higher in the PRP group, confirming the great rgenerative potential of this type of treatment. In this sense, our results are consistent with those reported by Alió and coauthors (2007) who also observed a significant increase in the density of caliciform cells with PRP.

Finally, we evaluated with each type of treatment the level of correlation among the improvement achieved with them and baseline parameters. In the PRP group, several weak correlations were found revealinga trend to a more degree of improvement in several parameters in those eyes with a more severe stage of the disease. Concerning moderate and strong correlations found, more visual improvement with PRP was 
associated with more level of visual disability at baseline. Furthermore, more significant improvements in the level of hyperemia, osmolarity, and corneal and conjunctival staining were associated with higher baseline levels of these variables. In contrast, these moderate and strong correlations were not found in the $\mathrm{SH}$ group which suggests that PRP may be a better option of treatment in those eyes with moderate and severe dry eye. Only in PRP group, more improvement in CIC outcome was associated with higher baseline levels of visual loss, hyperemia, and corneal and conjunctival staining.

In conclusion, PRP treatment in hyposecretory dry eye induces a positive effect over symptomatology and different physiopathological mechanisms responsable for this condition, and leads to a better restoration of the homeostasis of the ocular surface than $0.18 \%$ sodium hyaluronate eye drops. Specifically, PRP allows a more significant visual improvement, better control of hiperemia and corneal and conjunctival staining, more relevant reduction of symptomatology and more efficient restoration of physiological levels of tear osmolarity and caliciform cell density than artificial tears in hyposecretory dry eye. This restoration is more significant especially in those eyes with more significant baseline ocular surface and visual degradation, being possibly the most recomendable option in eyes with moderate and severe dry eye. Likewise, the beneficial effect of PRP is higher during the first 15 days of treatment. 


\section{REFERENCES}

Alió JL, Abad M, Artola A (2007): Use of autologous platelet-rich plasma in the treatment of dormant corneal ulcers. Ophthalmology 114: 1286-93.

Alio JL, Colecha JR, Pastor S, Rodriguez A, Artola A (2007): Symptomatic dry eye treatment with autologous platelet-rich plasma. Ophthalmic Res 39: 124-9.

Alió JL, Rodriguez AE (2013): Bovine pericardium membrane (tutopatch) combined with solid platelet-rich plasma for the management of perforated corneal ulcers. Cornea 32: 619-24.

Alio JL, Rodriguez AE (2015): Eye platelet-rich plasma in the treatment of ocular surface disorders. Curr Opin Ophthalmol 26: 325-32.

Alio JL, Rodriguez AE, Abdelghany AA, Oliveira RF (2017): Autologous platelet-rich plasma eye drops for the treatment of post-LASIK chronic ocular surface syndrome. J Ophthalmol 2017: 2457620.

Amparo F, Wang H (2013): The Ocular Redness Index: a novel automated method for measuring ocular injection. Invest Ophthalmol Vis Sci 54: 4821-6.

Anitua E, Muruzabal F (2016): Plasma rich in growth factors for the treatment of ocular surface diseases. Curr Eye Res 41: 875-82.

Anitua E (2015): Autologous serum and plasma rich in growth factors in ophthalmology: preclinical and clinical studies. Acta Ophthalmol 93: e605-14.

Avila MY (2014): Restoration of human lacrimal function following plateletrich plasma injection. Cornea 33: 18-21.

Baudouin C, Barton K, Cucherat M, Traverso C (2015): The measurement of bulbar hyperemia: challenges and pitfalls. Eur J Ophthalmol 25: 273-279. 
Benítez-Del-Castillo J, Labetoulle M, Baudouin C, Rolando M, Akova YA, Aragona P, Geerling G, Merayo-Lloves J, Messmer EM, Boboridis K (2017): Visual acuity and quality of life in dry eye disease: Proceedings of the OCEAN group meeting. Ocul Surf 15: 169-178.

Bron AJ, de Paiva CS, Chauhan SK, Bonini S, Gabison EE, Jain S, Knop E, Markoulli M, Ogawa Y, Perez V, Uchino Y, Yokoi N, Zoukhri D, Sullivan DA (2017): TFOS DEWS II pathophysiology report. Ocul Surf 15: 438-510.

Bron AJ, Evans VE, Smith JA (2003): Grading of corneal and conjunctival staining in the context of other dry eye tests. Cornea 22: 640-50.

Celebi AR, Ulusoy C, Mirza GE (2014): The efficacy of autologous serum eye drops for severe dry eye syndrome: a randomized double-blind crossover study. Graefes Arch Clin Exp Ophthalmol 252: 619-26.

del Castillo JM, de la Casa JM, Sardiña RC, Fernández RM, Feijoo JG, Gómez AC, Rodero MM, Sánchez JG (2002): Treatment of recurrent corneal erosions using autologous serum. Cornea 21: 781-3.

Drew VJ, Tseng CL, Seghatchian J, Burnouf T (2018): Reflections on dry eye syndrome treatment: therapeutic role of blood products. Front Med (Lausanne) 5: 33.

Egbert PR, Lauber S, Maurice DM (1977): A simple conjunctival biopsy. Am J Ophthalmol 84: 798-801.

Fox RI, Chan R, Michelson JB (1984): Beneficial effect of artificial tears made with autologous serum in patients with keratoconjunctivitis sicca. Arthritis Rheum 27: $459-61$.

Goto E, Shimmura S, Shimazaki J, Tsubota K (2001): Treatment of superior limbic keratoconjunctivits by appication of autologus serum. Cornea 20: 807-810. 
International Dry Eye Workshop (2007) The definition and classification of dry eye disease. In: 2007 Report of the International Dry Eye Workshop (DEWS). Ocul Surf 5: 75-92.

Javaloy J, Alio JL, Rodriguez AE, Vega A, Munoz G (2013): Effect of plateletrich plasma in nerve regeneration after LASIK. J Refract Surg 29: 213-9.

Jones L, Downie LE, Korb D, Benitez-Del-Castillo JM, Dana R, Deng SX, Dong PN, Geerling G, Hida RY, Liu Y, Seo KY, Tauber J, Wakamatsu TH, Xu J, Wolffsohn JS, Craig JP (2017): TFOS DEWS II Management and Therapy Report. Ocul Surf 15: 575-628.

Kaido M, Matsumoto Y, Shigeno Y, Ishida R, Dogru M, Tsubota K (2011): Corneal fluorescein staining correlates with visual function in dry eye patients. Invest Ophthalmol Vis Sci 52: 9516-22.

Kojima T, Ishida R, Dogru M, Goto E, Matsumoto Y, Kaido M, Tsubota K (2005): The effect of autologous serum eyedrops in the treatment of severe dry eye disease: a prospective randomized case-control study. Am J Ophthalmol 139: 242-6.

Lee JH, Kim MJ, Ha SW, Kim HK (2016): Autologous platelet-rich plasma eye drops in the treatment of recurrent corneal erosions. Korean J Ophthalmol 30: 101-7.

López-Miguel A, Tesón M, Martín-Montañez V, Enríquez-de-Salamanca A, Stern ME, González-García MJ, Calonge M (2016) Clinical and molecular inflammatory response in Sjögren syndrome-associated dry eye patients under desiccating stress. Am J Ophthalmol 161: 133-41.

López-Plandolit S, Morales MC, Freire V, Grau AE, Durán JA (2011): Efficacy of plasma rich in growth factors for the treatment of dry eye. Cornea 30: 1312-7. 
Marquez De Aracena Del Cid R (2009): Subconjunctival application of regenerative factor-rich plasma for the treatment of ocular alkali burns. Eur $\mathrm{J}$ Ophthalmol 19: 909-15.

Matsumoto Y, Dogru M, Goto E, Ohashi Y, Kojima T, Ishida R, Tsubota K (2004): Autologus serum application in the treatment of neurotrophic keratopathy. Ophthalmology 111: 1115-1120.

Nelson JD, Wright JC (1984): Conjunctival goblet cell densities in ocular surface disease. Arch Ophthalmol 102: 1049-51.

Nishida T, Nakamura M (1996): Sinergistics effects of substance P with insulin like growth factor-1 on epitelial migration of the cornea. J Cell Phisiol 169: 159-66.

Noble BA, Loh RS (2004): Comparison of autologous serum eye drops with conventional therapy in a randomised controlled crossover trial for ocular surface disease. Br J Ophthalmol 88: 647-52.

Noda-Tsuruya T, Asano-Kato N (2006): Autologous serum eye drops for dry eye after LASIK. J Refract Surg 22: 61-6.

Panda A, Jain M, Vanathi M, Velpandian T, Khokhar S, Dada T (2012): Topical autologous platelet rich plasma eyedrops for acute corneal chemical injury. Cornea 31: 989-93.

Pezzotta S, Del Fante C (2012): Autologous platelet lysate for treatment of refractory ocular GVHD. Bone Marrow Transplant 47: 1558-63.

Ralph RA, Doane MG (1975): Clinical experience with a mobile ocular perfusion pump. Arch Ophthalmol 93: 1039-43.

Ronci C, Ferraro AS (2015): Platelet-rich plasma as treatment for persistent ocular epithelial defects. Transfus Apher Sci 52: 300-4. 
Sangwan VS, Zafirakis P, Foster CS (1997): Mooren's ulcer: current concepts in management. Indian J Ophthalmol 45: 7-17.

Tananuvat N, Daniell M (2001): Controlled study of the use of autologous serum in dry eye patients. Cornea 20: 802-6.

Thulasi P, Djalilian AR (2017): Update in current diagnostics and therapeutics of dry eye disease. Ophthalmology 124(11S): S27-S33.

Urzua CA, Vasquez DH, Huidobro A, Hernández H, Alfaro J (2012): Randomized double-blind clinical trial of autologous serum versus artificial tears in dry eye syndrome. Curr Eye Res 37: 684-8.

Wroblewski AP, Melia HJ, Wright VJ (2010): Application of platelet-rich plasma to enhance tissue repair. Oper Tech Orthop 20: 98-105. 


\section{Figure legends}

Figure 1.- Changes in OSDI score during the follow-up in PRP (grey bars) and SH groups (white bars). Percentage of change is also displayed.

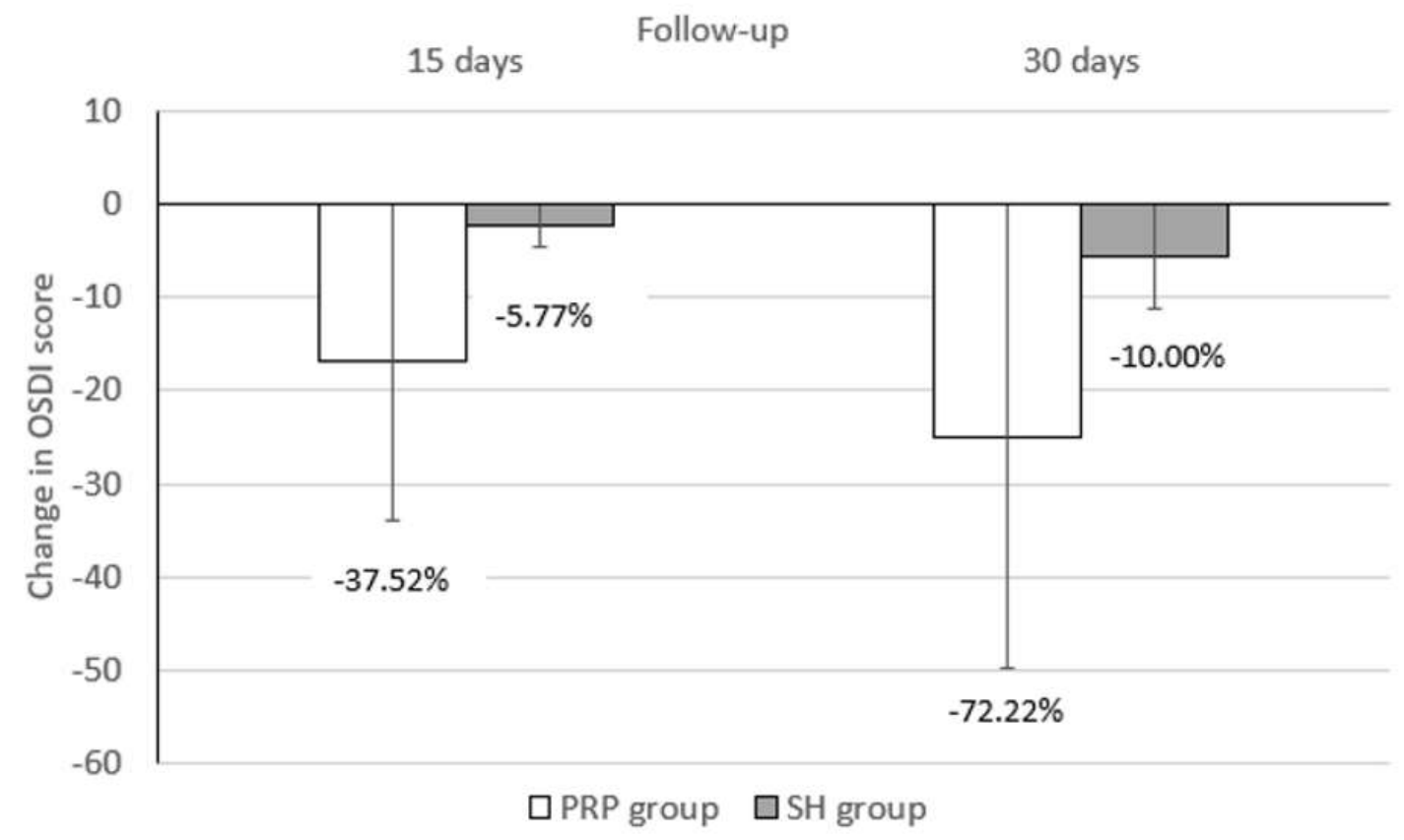


Figure 2.- Changes in tear osmolarity during the follow-up in PRP (grey bars) and SH groups (white bars). Percentage of change is also displayed.

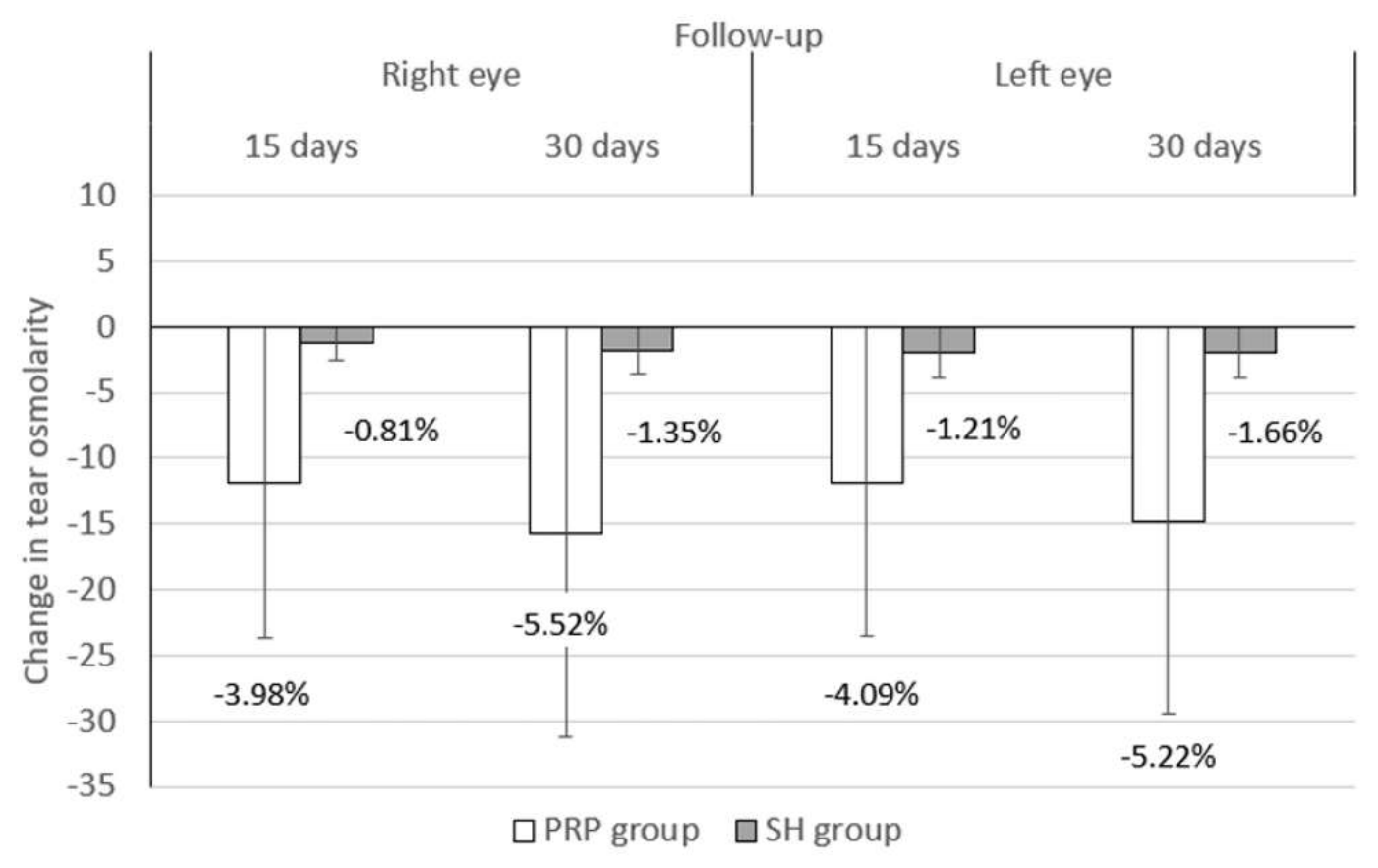




\begin{tabular}{|c|c|c|c|}
\hline \multicolumn{4}{|l|}{ Median (Range) } \\
\hline \multirow[t]{2}{*}{ Age (years) } & $62.1(11.2)$ & $66.2(11.0)$ & 0.097 \\
\hline & $64.0(36$ a 84$)$ & $66.0(36$ a 90$)$ & \\
\hline \multirow[t]{2}{*}{ OSDI score } & $59.02(17.86)$ & $52.82(19.74)$ & 0.204 \\
\hline & $60.00(23$ a 93$)$ & $54.00(10$ a 89$)$ & \\
\hline \multirow[t]{2}{*}{ Decimal CDVA RE } & $0.64(0.27)$ & $0.75(0.21)$ & 0.115 \\
\hline & $0.70(0.1$ a 1.0$)$ & $0.80(0.3$ a 1.0$)$ & \\
\hline \multirow[t]{2}{*}{ Decimal CDVA LE } & $0.65(0.26)$ & $0.74(0.23)$ & 0.168 \\
\hline & $0.70(0.1$ a 1.0$)$ & $0.75(0.30$ a 1.0$)$ & \\
\hline \multirow[t]{2}{*}{ Hyperemia RE } & $2.7(0.7)$ & $2.3(0.6)$ & 0.002 \\
\hline & $3.0(1 \mathrm{a} 4)$ & $2.0(1 \mathrm{a} 4)$ & \\
\hline \multirow[t]{2}{*}{ Hyperemia LE } & $2.7(0.6)$ & $2.2(0.6)$ & 0.001 \\
\hline & $3.0(2$ a 4$)$ & $2.0(1 \mathrm{a} 4)$ & \\
\hline \multirow[t]{2}{*}{ Osmolarity RE (mOsm/l) } & $314.67(18.16)$ & $311.97(7.56)$ & 0.886 \\
\hline & $312.00(285$ a 376$)$ & $313.00(285$ a 322$)$ & \\
\hline \multirow[t]{2}{*}{ Osmolarity LE (mOsm/l) } & $314.02(17.24)$ & $311.71(6.79)$ & 0.424 \\
\hline & $315.00(285$ a 370$)$ & $313.00(287$ a 320$)$ & \\
\hline \multirow[t]{2}{*}{ Schirmer RE (mm) } & $3.74(1.76)$ & $4.71(1.06)$ & 0.022 \\
\hline & $4.00(1 \mathrm{a} 6)$ & $5.00(1 \mathrm{a} 6)$ & \\
\hline \multirow[t]{2}{*}{ Schirmer LE (mm) } & $4.05(1.85)$ & $4.40(1.12)$ & 0.789 \\
\hline & $5.00(0$ a 6$)$ & $4.00(1 \mathrm{a} 6)$ & \\
\hline \multirow[t]{2}{*}{ TF-BUT RE (s) } & $5.6(1.1)$ & $5.6(0.9)$ & 0.769 \\
\hline & $6.0(3$ a 7$)$ & $5.0(4$ a 8$)$ & \\
\hline \multirow[t]{2}{*}{ TF-BUT LE (s) } & $5.5(1.2)$ & $5.7(1.3)$ & 0.542 \\
\hline & $6.0(3$ a 7$)$ & $6.0(2$ a 9$)$ & \\
\hline \multirow[t]{2}{*}{ Conjunctival staining RE } & $3.1(1.3)$ & $1.7(1.1)$ & $<0.001$ \\
\hline & $3.0(1$ a 6$)$ & $2.0(0$ a 4$)$ & \\
\hline \multirow[t]{2}{*}{ Conjunctival staining LE } & $2.9(1.2)$ & $1.7(1.3)$ & $<0.001$ \\
\hline & $3.0(1$ a 6$)$ & $1.0(0$ a 6$)$ & \\
\hline \multirow[t]{2}{*}{ Corneal staining RE } & $3.0(1.2)$ & $1.8(1.0)$ & $<0.001$ \\
\hline & $3.0(1$ a 5$)$ & $2.0(1$ a 5$)$ & \\
\hline \multirow[t]{2}{*}{ Corneal staining LE } & $2.8(1.2)$ & $1.7(1.0)$ & $<0.001$ \\
\hline & $3.0(1$ a 5$)$ & $1.0(0$ a 5$)$ & \\
\hline \multirow{2}{*}{$\begin{array}{c}\text { Caliciform cell density } \\
\text { measured by CIC (cel/mm²) }\end{array}$} & $198.29(111.28)$ & $335.73(125.87)$ & 0.007 \\
\hline & $144.50(36$ a 390$)$ & $320.00(24$ a 537$)$ & \\
\hline
\end{tabular}

Table 1.- Baseline values of the parameters evaluated in the two groups of the current series: sodium hyaluronate (SH) and platelet rich plasma (PRP) groups. Abbreviations: SD, standard deviation; CDVA, corrected distance visual acuity; RE, right eye; LE, left eye; TF-BUT, tear film break-up time; CIC, conjunctival impression cytology. 


\begin{tabular}{|c|c|c|c|}
\hline $\begin{array}{c}\text { Mean (SD) } \\
\text { Median (Range) }\end{array}$ & PRP & SH & p-value \\
\hline OSDI score & $\begin{array}{l}-16.93(12.71) \\
-15.00(-55 \text { a } 4)\end{array}$ & $\begin{array}{c}-2.28(5.49) \\
-2.00(-18 \text { a } 13)\end{array}$ & 0.001 \\
\hline Decimal CDVA RE & $\begin{array}{c}0.11(0.11) \\
0.10(0.00 \text { a } 0.40)\end{array}$ & $\begin{array}{c}0.01(0.04) \\
0.00(-0.10 \text { a } 0.10)\end{array}$ & 0.001 \\
\hline Decimal CDVA LE & $\begin{array}{c}0.12(0.13) \\
0.10(-0.10 \text { a } 0.60)\end{array}$ & $\begin{array}{c}0.01(0.08) \\
0.00(-0.10 \text { a } 0.30)\end{array}$ & $<0.001$ \\
\hline Hyperemia RE & $\begin{array}{c}-1.10(0.72) \\
-1.00(-2.00 \text { a } 0.00)\end{array}$ & $\begin{array}{c}-0.11(0.39) \\
0.00(-2.00 \text { a } 0.00)\end{array}$ & 0.001 \\
\hline Hyperemia LE & $\begin{array}{c}-1.16(0.78) \\
-1.00(-2.00 \text { a } 0.00)\end{array}$ & $\begin{array}{c}-0.03(0.30) \\
0.00(-1.00 \text { a } 1.00)\end{array}$ & $<0.001$ \\
\hline $\begin{array}{l}\text { Osmolarity RE } \\
(\text { mOsm/l) }\end{array}$ & $\begin{array}{l}-11.87(12.73) \\
-8.00(-56 \text { a } 2)\end{array}$ & $\begin{array}{c}-1.29(3.31) \\
-1.50(-12 \text { a } 7)\end{array}$ & 0.002 \\
\hline $\begin{array}{c}\text { Osmolarity LE } \\
\text { (mOsm/l) }\end{array}$ & $\begin{array}{l}-11.79(12.86) \\
-8.00(-51 \text { a } 8)\end{array}$ & $\begin{array}{l}-1.94(4.66) \\
0.00(-12 \text { a } 5)\end{array}$ & 0.003 \\
\hline Schirmer RE (mm) & $\begin{array}{c}1.26(1.19) \\
1.00(-2 \text { to } 5)\end{array}$ & $\begin{array}{l}0.37(0.91) \\
0.00(-1 \text { a } 2)\end{array}$ & $<0.001$ \\
\hline Schirmer LE (mm) & $\begin{array}{l}1.42(1.79) \\
1.00(-3 \text { a } 7)\end{array}$ & $\begin{array}{l}0.31(0.96) \\
0.00(-3 \text { a } 2)\end{array}$ & 0.002 \\
\hline TF-BUT RE (s) & $\begin{array}{c}0.3(0.6) \\
0.0(-1 \text { a } 1)\end{array}$ & $\begin{array}{c}0.2(0.5) \\
0.0(-1 \text { a } 1)\end{array}$ & 0.200 \\
\hline TF-BUT LE (s) & $\begin{array}{c}0.6(0.7) \\
1.0(-1 \text { a } 2)\end{array}$ & $\begin{array}{c}0.0(0.7) \\
0.0(-2 \text { a } 1)\end{array}$ & 0.003 \\
\hline $\begin{array}{c}\text { Conjunctival staining } \\
\text { RE }\end{array}$ & $\begin{array}{c}-1.9(1.2) \\
-2.0(-5 \text { a } 1)\end{array}$ & $\begin{array}{c}-0.2(0.5) \\
0.0(-2 \text { a } 0)\end{array}$ & 0.001 \\
\hline $\begin{array}{c}\text { Conjunctival staining } \\
\text { LE }\end{array}$ & $\begin{array}{c}-1.8(1.0) \\
-2.0(-5 \text { a } 0)\end{array}$ & $\begin{array}{l}-0.3(0.7) \\
0.0(-4 \text { a } 0)\end{array}$ & 0.001 \\
\hline Corneal staining RE & $\begin{array}{c}-1.8(0.9) \\
-2.0(-5 \text { a } 0)\end{array}$ & $\begin{array}{c}-0.2(0.5) \\
0.0(-1 \text { a } 1)\end{array}$ & $<0.001$ \\
\hline Corneal staining LE & $\begin{array}{c}-1.9(1.0) \\
-2.0(-5 \text { a } 0)\end{array}$ & $\begin{array}{c}-0.1(0.3) \\
0.0(-1 \text { a } 0)\end{array}$ & $<0.001$ \\
\hline
\end{tabular}

Table 2.- Changes occurring in the different clinical parameters evaluated 15 days after beginning with the treatment in the two groups of the current series: sodium hyaluronate $(\mathrm{SH})$ and platelet rich plasma (PRP) groups. Abbreviations: SD, standard deviation; CDVA, corrected distance visual acuity; RE, right eye; LE, left eye; TF-BUT, tear film break-up time. 


\begin{tabular}{|c|c|c|c|}
\hline $\begin{array}{c}\text { Mean (SD) } \\
\text { Median (Range) }\end{array}$ & PRP & SH & p-value \\
\hline OSDI score & $\begin{array}{l}-24.86(15.71) \\
-21.50(-66 \mathrm{a}-4)\end{array}$ & $\begin{array}{c}-5.56(5.67) \\
-5.00(-20 \text { a } 8)\end{array}$ & 0.001 \\
\hline Decimal CDVA RE & $\begin{array}{c}0.15(0.16) \\
0.10(0.00 \text { a } 0.70)\end{array}$ & $\begin{array}{c}0.03(0.08) \\
0.00(-0.10 \text { a } 0.40)\end{array}$ & 0.004 \\
\hline Decimal CDVA LE & $\begin{array}{c}0.16(0.16) \\
0.10(0.00 \text { a } 0.60)\end{array}$ & $\begin{array}{c}0.03(0.08) \\
0.00(-0.10 \text { a } 0.30)\end{array}$ & 0.002 \\
\hline Hyperemia RE & $\begin{array}{c}-1.3(0.7) \\
-1.0(-3 \text { a } 0)\end{array}$ & $\begin{array}{l}-0.2(0.5) \\
0.0(-2 \text { a } 1)\end{array}$ & 0.002 \\
\hline Hyperemia LE & $\begin{array}{c}-1.3(0.7) \\
-1.0(-3 \text { a } 0)\end{array}$ & $\begin{array}{c}-0.1(0.4) \\
0.0(-1 \text { a } 1)\end{array}$ & 0.002 \\
\hline Osmolarity RE (mOsm/l) & $\begin{array}{l}-15.64(15.48) \\
-13.00(-55 \text { a } 7)\end{array}$ & $\begin{array}{c}-1.82(6.83) \\
-2.00(-12 \text { a } 31)\end{array}$ & 0.002 \\
\hline Osmolarity LE (mOsm/l) & $\begin{array}{l}-14.74(14.44) \\
-11.00(-51 \text { a } 7)\end{array}$ & $\begin{array}{c}-1.91(7.24) \\
-2.00(-17 \text { a } 23)\end{array}$ & 0.002 \\
\hline Schirmer RE (mm) & $\begin{array}{c}1.9(1.5) \\
2.0(0 \mathrm{a} 7)\end{array}$ & $\begin{array}{c}0.3(1.2) \\
0.0(-2 \text { a } 2)\end{array}$ & 0.002 \\
\hline Schirmer LE (mm) & $\begin{array}{c}1.9(2.1) \\
1.0(-2 \text { a } 7)\end{array}$ & $\begin{array}{c}0.5(1.3) \\
1.0(-3 \text { a } 3)\end{array}$ & 0.001 \\
\hline TF-BUT RE (s) & $\begin{array}{c}0.6(0.8) \\
1.0(-1 \text { a } 2)\end{array}$ & $\begin{array}{c}0.3(0.8) \\
0.0(-2 \text { a } 2)\end{array}$ & 0.102 \\
\hline TF-BUT LE (s) & $\begin{array}{c}0.7(0.8) \\
1.0(-1 \text { a } 3)\end{array}$ & $\begin{array}{c}0.2(0.9) \\
0.0(-2 \text { a } 2)\end{array}$ & 0.024 \\
\hline Conjunctival staining RE & $\begin{array}{c}-2.5(1.1) \\
-2.0(-5 \text { a }-1)\end{array}$ & $\begin{array}{c}-0.3(0.6) \\
0.0(-2 \text { a } 0)\end{array}$ & $<0.001$ \\
\hline Conjunctival staining LE & $\begin{array}{c}-2.2(0.9) \\
-2.0(-5 \mathrm{a}-1)\end{array}$ & $\begin{array}{l}-0.3(0.8) \\
0.0(-4 \text { a } 0)\end{array}$ & 0.001 \\
\hline Corneal staining RE & $\begin{array}{c}-2.3(1.1) \\
-2.0(-5 \text { a } 1)\end{array}$ & $\begin{array}{l}-0.3(0.5) \\
0.0(-1 \text { a } 0)\end{array}$ & $<0.001$ \\
\hline Corneal staining LE & $\begin{array}{c}-2.3(0.9) \\
-2.0(-5 \mathrm{a}-1)\end{array}$ & $\begin{array}{l}-0.2(0.5) \\
0.0(-2 \text { a } 0)\end{array}$ & 0.001 \\
\hline $\begin{array}{c}\text { Caliciform cell density } \\
\text { measured by CIC }(\text { cel/mm²) }\end{array}$ & $\begin{array}{c}293.71(100.05) \\
291.00(89 \text { a } 455)\end{array}$ & $\begin{array}{l}45.60(70.21) \\
28.0(1 \text { a } 293)\end{array}$ & 0.004 \\
\hline
\end{tabular}

Table 3.- Changes occurring in the different clinical parameters evaluated 30 days after beginning with the treatment in the two groups of the current series: sodium hyaluronate $(\mathrm{SH})$ and platelet rich plasma (PRP) groups. Abbreviations: SD, standard deviation; CDVA, corrected distance visual acuity; RE, right eye; LE, left eye; TF-BUT, tear film break-up time; CIC, conjunctival impression cytology. 